\title{
Importance of Polymorphism in Improving the Potency of Bioactive Molecules
}

\author{
Anil Kumar, Jyoti Chauhan, Kshatresh Dutta Dubey, Subhabrata Sen and Parthapratim Munshi* \\ Department of Chemistry, School of Natural Science, Shiv Nadar University, Tehsil Dadri, Uttar Pradesh- 201314, India \\ parthapratim.munshi@snu.edu.in
}

Thiazolidinone derivatives play crucial roles in anticancer (breast cancer, lung cancer and leukaemia) drug discovery process. ${ }^{1}$ Especially, 5-arylidene-2-aminothiazolidinones are found to show antimitotic activities against MCF7 breast cancer cells. ${ }^{2}$ However, bioactive molecules are known to undergo polymorphic modifications under certain conditions and polymorphs often exhibit distinct physicochemical and biopharmaceutical properties. ${ }^{3}$ Indeed, polymorphism has been the critical issue in drug development process. ${ }^{4}$ Therefore, systematic characterization of polymorphism in bioactive molecules is highly essential. Here, we report the discovery of polymorphism on 5-arylidene-2-aminothiazolidinones derivatives and their systematic characterizations via single-crystal X-ray diffraction, thermal analyses and spectroscopic analysis. The estimation of energies in terms of interaction energies, lattice energies and energy frameworks bring out the energetic stabilities of the polymorphs. Solubility, dissolution rate and phase stability experiments confirm that the thermodynamically most stable form exist with least solubility and dissolution rate. Further, we investigated the extent of inhibition imposed by our library of polymorphs on the proliferation of MCF7 breast cancer cell lines and also the extent of their binding to the target enzyme ( $\gamma$-enolase). Our preliminary cellular assay suggests that in general specific polymorphic forms are indeed more potent than the corresponding bulk form. The $\gamma$-enolase binding assay also demonstrated a trend similar to that of the phenotypic screening against MCF7. Furthermore, the binding affinity of the polymorphs with $\gamma$-enolase as estimated via molecular dynamic simulations is in well agreement with the binding assay results. The aforementioned experiments in general emphasized the importance of polymorphism in improving the biological potency of the molecules. We believe that the studies of this kind would help screening potent drug molecules in pharmaceutical industries.

[1] R. B. Lesyk and B. S. Zimenkovsky, Curr. Org. Chem., 2004, 8, 1547-1577

[2] C. Bathula, S. tripathy, R. Srinivasan, K. k. Jha, A. Ganguly, G. Chakraboarti, S. Singh, P. Munshi and S. Sen, Org. Biomol. Chem., 2016, 14, 80538063.

[3] J. Bernstein, Polymorphism in Molecular Crystals, Oxford University Press Inc., New York, 2002.

[4] Jeong et al. J. Pharm. Invest. 2010

Keywords: Bioactive Molecules, Polymorphism; Phenotypic Screening, Binding Assay, MD simulation. 